\title{
Fast Median Computation For Symmetric, Orthogonal Matrices Under the Rank Distance
}

\author{
Joao Meidanis ${ }^{\mathrm{a}}$, Leonid Chindelevitch ${ }^{\mathrm{b}}$ \\ ${ }^{a}$ University of Campinas, Campinas, Brazil \\ ${ }^{b}$ Imperial College, London, United Kingdom
}

\begin{abstract}
Biological genomes can be represented as square, symmetric, orthogonal, 0-1 matrices. It turns out that the rank distance applied to two genome matrices has a biological significance: it is related to the smallest number of basic rearrangement mutations, such as reversals, translocations, transpositions (taken with weight 2), etc. that explain the differences between the two genomes. Therefore, closer genomes will produce smaller rank distances.

An important tool in this context is the median problem: given three genomes $A, B$, and $C$, find a fourth genome $M$ that minimizes $d(A, M)+$ $d(B, M)+d(C, M)$. For genome matrices, the computational complexity of this problem is currently unknown. However, for orthogonal matrices, there are fast algorithms that solve it exactly.

One such algorithm uses a "walk towards the median" paradigm. Starting from any of the input matrices, say, $B$, the algorithm produces rank-1 "steps", which are rank-1 matrices that, added to $B$, decrease its rank distance to both $A$ and $C$ simultaneously. It can be shown that such steps always exist for orthogonal matrices, and can be found in polynomial time. The algorithm stops when no more improvement can be made, which is equivalent to saying that $B$ lies between $A$ and $C$ in terms of the rank distance (the triangle inequality becomes an equality). There is an $O\left(n^{\omega+1}\right)$ algorithm implementing this idea, where $\omega$ is the matrix multiplication exponent. Here we propose a novel scheme that works for symmetric orthogonal matrices, and produces a median, also guaranteed to be symmetric, in $O\left(n^{\omega}\right)$ time.

There is another $O\left(n^{\omega}\right)$ time algorithm that produces the so-called $M_{I}$
\end{abstract}

Email addresses: meidanis@ic.unicamp.br (Joao Meidanis), lchindel@ic.ac.uk (Leonid Chindelevitch) 
median, which agrees with the majority in the subspaces where $A=B$, $B=C$, or $C=A$, and is equal to the identity in the orthogonal complement of the sum of these subspaces. However, this algorithm produces a different median, and has only been proved to be correct for genomic matrices. The algorithm we present here is more general.

Keywords:

Eigenvalues, singular values, and eigenvectors, Orthogonal matrices, Hermitian, skew-Hermitian, and related matrices 2020 MSC: 15A18, 15B10, 15B57

\section{Introduction}

Genomes evolve by accumulating mutations that get fixed into their DNA and passed on to their descendants. Mutations may involve only one or a few consecutive DNA nucleotides, as in an insertion, deletion, and substitution of a few monomers in a large DNA molecule. Other mutations include the movement, incorporation, or deletion of large chunks of the genome, including entire chromosomes, and these are called genome rearrangements. Examples of such rearrangements are inversions, transpositions, translocations, and chromosome fusions and fissions.

For a complete evolutionary analysis it is important to consider all types of mutations. However, in some circumstances, genome rearrangements are more prevalent, and significant results can be obtained by taking into account only this kind of mutation. For instance, the evolution of mitochondrial DNA (mtDNA) in plants seems to proceed much more by means of shuffling genes around in larger blocks than by local mutations involving a small number of nucleotides. Palmer and Hebron studied physical maps of six Brassica mtDNAs, and estimated that, at the DNA level, mitochondrial DNA is $99.9 \%$ conserved, whereas the order and orientation of genes changes much more rapidly [1].

There seem to be other relevant reasons to study genome rearrangements [2]. In bacteria, these rearrangements can be related to adaptation to new niches [3], and may be implicated in acquired antibiotic resistance [4]. Some types of rearrangements may be preferred, such as those moving groups of genes to the same type of strand (lagging or leading strand) [3]. In mammalian genomes, it has been observed that rearrangements usually involve breakpoints that are spatially close; this may indicate that there is selec- 
tion against rearrangements that act on spatially distant breakpoints [5]. In a more refined analysis, it is common to add restrictions on the types of breakpoints allowed [6].

To model genome rearrangements as mathematical objects in order to process them, many approaches are possible, depending on the type of genomes and operations one wants to include. For instance, genomes can have linear or circular chromosomes, or a mixture of the two. Operations may involve just one kind, such as an inversion, or multiple kinds. In general, the more complex a model is, the more difficult it is to solve the relevant problems in this model. One has to strike a balance, allowing for enough complexity to render the results biologically relevant, while still being able to use fast algorithms in the processing of the data. In this paper, we opt for the rank distance model, where genomes are represented by large, square matrices and the rank distance measures their evolutionary distance.

Our goal in this paper is to show how to speed up the so-called orthogonal algorithm [7] for finding the generalized median of three genomes according to the rank distance [8]. In this algorithm, a series of Householder transformations are applied to one of the input matrices, resulting in a "walk" towards the median. Each step takes $O\left(n^{\omega}\right)$ time, where $\omega$ is the matrix multiplication exponent, since it involves solving a system of linear equations, yielding an $O\left(n^{\omega+1}\right)$ algorithm in the worst case, since $O(n)$ steps are needed to complete the walk in the worst case. The main idea here is to find conditions under which multiple Householder transformations can be applied simultaneously, and to leverage those conditions to speed up the walk towards the median by taking multiple steps at a time. We end up with an algorithm that needs just 2 steps, decreasing the time complexity to $O\left(n^{\omega}\right)$.

The rest of this paper is organized as follows. In Section 2 we present the basic definitons regarding the model used and discuss its biological relevance. Scetion 3 contains our main results, which improve the complexity of the orthogonal algorithm for medians of orthogonal, symmetric matrices. A partial generalization to permutation matrices, not necessarily genomic, is studied in Section 4. Finally, our concluding remarks and open questions are presented in Section 5 . 


\section{Background}

\subsection{Representing Genomes as Matrices}

In a series of recent papers $[8,7,9,10,11]$, our group has proposed the representation of genomes as square, $0-1$, sparse matrices and the use of rank distance to study genome evolution by large-scale events. Our matrix representation relies on a previous step consisting of the definition of homologous or evolutionarily corresponding regions in the genomes being compared. Usually, this entails the identification of the genes in each genome of interest and an assessment of which genes in one genome correspond to which genes in the other genomes. However, other conserved regions, such as bacterial operons (which are consecutive genes that are jointly expressed and contribute to the same function) or syntenic regions (regions containing groups of genes with similar sequence and function in different species) can be used. These syntenic regions can be the 13 conserved segments between human and mouse identified by Nadeau and Taylor in a seminal 1984 paper [12], or the 7 conserved regions between the $\mathrm{X}$ chromosome of human and mouse identified more recently by Pevzner and Tesler [13], to name a few well-known instances.

While other genome comparison methods require that the conserved segments in each one of two genomes being compared be in a bijective relationship, our scheme allows for regions that exist in only one or a few of the genomes being compared. Ideally, a method should also support repeated regions in the same genome, and we plan to extend the scheme to support this, as has been done for other models [14].

Our current model works as follows. Let $G$ be a set of genomes of interest and $R$ a set of conserved genome regions that contains all the regions of interest in the genomes of $G$. In general, no genome in $G$ will contain all the regions in $R$, but for every genome $g \in G$, all its regions will belong to $R$. We assume that each region appears at most once in a given genome $g \in G$. See Figure 1 for examples.

A genome is a set of chromosomes. Each chromosome is a linear succession of consecutive regions from $R$. However, due to the double helix structure of DNA molecules, a region $a$ may appear as itself or as its reverse complement, denoted by $\bar{a}$, in a chromosome. Thus, if $a, b, c$ are regions in our chromosome, the possible chromosomes are $\left[\begin{array}{lll}a & b & c\end{array}\right],\left[\begin{array}{lll}a & \bar{b} & c\end{array}\right],\left[\begin{array}{lll}b & \bar{a} & c\end{array}\right]$, $\left[\begin{array}{lll}c & \bar{b} & a\end{array}\right]$, etc. There are $r ! 2^{r}$ combinations for $r$ regions. However, due to the same DNA structure, a combination and its reverse complement are the same 

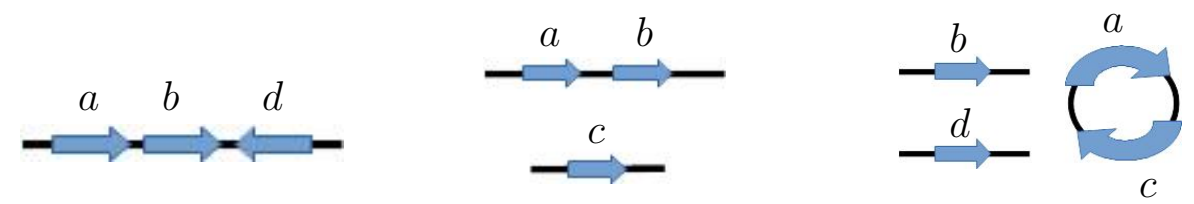

Figure 1: Genomes over region set $R=\{a, b, c, d\}$. The leftmost genome has just one linear chromosome and does not have region $c$. The middle genome has two linear chromosomes and does not have region $d$. The rightmost genome has three chromosomes, two linear and one circular, and has all regions in $R$.

chromosome. Thus $\left[\begin{array}{lll}a & b & \bar{c}\end{array}\right]$ and $\left[\begin{array}{lll}c & \bar{b} & \bar{a}\end{array}\right]$ correspond to the same chromosome. This reduces the total number of possible linear chromosomes with $r$ regions to $r ! 2^{r-1}$.

Chromosomes can also be circular, which means that there is an extra link between their first and last extremities. Circular chromosomes are represented with parentheses, as in $(a b \bar{c})$, instead of square brackets. Since they are circular, there is no starting gene, so the same chromosome can be written as $\left(\begin{array}{lll}b & \bar{c} & a\end{array}\right)$ or as $\left(\begin{array}{lll}\bar{c} & a & b\end{array}\right)$. On top of that, the reverse complement also corresponds to the same chromosome: $(c \bar{b} \bar{a}),(\bar{b} \bar{a} c),(\bar{a} c \bar{b})$. In all these representations the undirected links between consecutive regions are the same. The total number of circular chromosomes with $r$ regions is thus $(r-1) ! 2^{r-1}$. The genomes in Figure 1 can be written as $\left\{\left[\begin{array}{lll}a & b & \bar{d}\end{array}\right]\right\}$ (leftmost genome), $\{[a b],[c]\}$ (middle genome), and $\{[b],(a c)\}$ (rightmost genome).

To represent a genome as a matrix, we need the unordered links between consecutive regions in the chromosomes. Every region $a$ has two extremities: its tail $a_{t}$ and its head $a_{h}$. A region can be considered an ordered entity, which is traversed from tail to head in biological processes. Therefore, the appearance of $a$ in a chromosome means extremities in the order $a_{t} a_{h}$, whereas $\bar{a}$ means the opposite order $a_{h} a_{t}$. For instance, chromosome $[\bar{a} \bar{b} c]$ has extremities appearing in the order $a_{h} a_{t} b_{h} b_{t} c_{t} c_{h}$ and the links between consecutive regions are $a_{t} b_{h}$ and $b_{t} c_{t}$. If we write the chromosome in its reverse complement form, that is, $\left[\begin{array}{lll}\bar{c} & b & a\end{array}\right]$, then the extremity order is $c_{h} c_{t} b_{t} b_{h} a_{t} a_{h}$ and the links are $c_{t} b_{t}$ and $b_{h} a_{t}$. Since the links are undirected, this results in the same links.

We are ready to define the matrix representation of a genome in $G$. Build a square matrix of dimension $2 r \times 2 r$, where $r$ is the number of regions, and choose an arbitrary but fixed order of all extremities of regions in $R$. We identify each extremity $x$ of a region in $R$ with the elementary column vector containing a 1 in the row corresponding to $x$ and a 0 everywhere else. 
Given a genome $g$, we represent each link $x y$ between two extremities in a chromosome of $g$ with the matrix $x y^{T}+y x^{T}$. If $x$ is a free end in $g$, i.e. it does not participate in any link, we represent it with $x x^{T}$. We get the matrix representation of $g$ by adding up all of these contributions; in other words:

$$
M(g)=\sum_{x y \text { link in } g}\left(x y^{T}+y x^{T}\right)+\sum_{x \text { free in } g} x x^{T} .
$$

Notice that $M(g)$ is a $0-1$, sparse, symmetric, square matrix for every $g \in G$. If $g$ contains all regions in $R$, then $M(g)$ is an orthogonal matrix. If $g$ lacks one or more regions from $R$, then $M(g)$ will have some rows and columns composed solely of zeros, and will not be orthogonal. However, in the rest of this paper we assume that the genomes being compared contain all the regions of $R$, so that we deal only with orthogonal matrices.

With respect to Figure 1 we have the following matrices for the first and last genomes:

$a_{t}$
$a_{h}$
$b_{t}$
$b_{h}$
$c_{t}$
$c_{h}$
$d_{t}$
$d_{h}$$\left[\begin{array}{llllllll}1 & 0 & 0 & 0 & 0 & 0 & 0 & 0 \\ 0 & 0 & 1 & 0 & 0 & 0 & 0 & 0 \\ 0 & 1 & 0 & 0 & 0 & 0 & 0 & 0 \\ 0 & 0 & 0 & 0 & 0 & 0 & 0 & 1 \\ 0 & 0 & 0 & 0 & 0 & 0 & 0 & 0 \\ 0 & 0 & 0 & 0 & 0 & 0 & 0 & 0 \\ 0 & 0 & 0 & 0 & 0 & 0 & 1 & 0 \\ 0 & 0 & 0 & 1 & 0 & 0 & 0 & 0\end{array}\right]\left[\begin{array}{llllllll}0 & 0 & 0 & 0 & 0 & 1 & 0 & 0 \\ 0 & 0 & 0 & 0 & 1 & 0 & 0 & 0 \\ 0 & 0 & 1 & 0 & 0 & 0 & 0 & 0 \\ 0 & 0 & 0 & 1 & 0 & 0 & 0 & 0 \\ 0 & 1 & 0 & 0 & 0 & 0 & 0 & 0 \\ 1 & 0 & 0 & 0 & 0 & 0 & 0 & 0 \\ 0 & 0 & 0 & 0 & 0 & 0 & 1 & 0 \\ 0 & 0 & 0 & 0 & 0 & 0 & 0 & 1\end{array}\right]$

\subsection{Biological Significance of the Rank Distance}

With genomes coded as matrices, we can use the rank distance $d(A, B)=$ $r(A-B)$ to provide a quantitative way of measuring their differences. To use the rank distance we need all the matrices to be of the same size, which is automatic if all the genome regions involved belong to the same region set $R$.

The rank distance has been studied in connection to codes [15]. Our confidence about its relevance in the context of evolutionary studies by large block mutations rests on the fact that all known large mutations frequently observed in real genomes yield genome pairs with very small rank distance, regardless of the size of the blocks involved. For instance, the matrices $A$, $B$ corresponding to two genomes differing by just an inversion will have $d(A, B)=2$, no matter how many conserved regions exist in the inverted 
block, and irrespective of the chromosome being linear or circular. This stems from the fact that only the links at the boundaries of the inverted region are affected. Likewise, a translocation leads to a distance of 2 , a transposition to a distance of 4 , and chromosome fission and fusion lead to a distance of 1 , all independently of the size of the blocks involved.

For two arbitrary genome matrices $A$ and $B$, there is always a series of basic operations, defined as operations yielding a distance of one or two, that can be applied to $A$ resulting in $B$ [16, Sec. 3.4]. Therefore, the rank distance can be interpreted as the minimum total weight of basic operations needed to explain the differences between $A$ and $B$, where the weight of a basic operation is the difference in rank between the genomes before and after it. In other words, the rank distance $d(A, B)$ may be seen as a measure of the amount of evolution separating the genomes corresponding to $A$ and $B$ with respect to large-scale operations.

\subsection{Reconstructing Evolutionary History and Ancestors}

Given pairwise distances between a set of genomes, or taxa in general, there are standard methods for constructing a phylogenetic tree which represents a hypothesis about the branching that occurred in the evolution of these taxa $[17,18]$. To estimate the genomes at the internal nodes of the tree, one widely used heuristic consists in starting with any assignment, usually one that is an easily obtained, such as an intermediate genome, i.e. a genome $C$ satisfying $d(A, C)+d(C, B)=d(A, B)$, between the two children $A$ and $B$ of each parent node, in a bottom-up fashion, and then gradually improving this assignment by considering every internal node in turn and its three neighbours, and looking for a replacement for the internal node genomes that locally improves the total branch length. To achieve this improvement, a problem called the Genome Median Problem must be solved.

The Genome Median Problem can be stated as follows. Given input genomes $A, B$, and $C$, find a genome $M$ that minimizes the total score, defined as

$$
s(M):=d(A, M)+d(B, M)+d(C, M) .
$$

Such a genome $M$ can replace the previous genome at the internal node, where $A, B, C$ are its three neighbors in the tree. If the original genome was not a median of $A, B$ and $C$, then replacing it by a median will locally improve the total branch length. Repeated application of this procedure to all internal nodes will gradually improve the total branch length. Even when 
a global minimum is not reached, this method tends to give good results in practice [19].

Although the complexity of the Genome Median Problem is unknown, there are polynomial-time algorithms to compute generalized medians, that is, matrices that minimize $s(M)$ but are not necessarily genomic: the $M_{I}$ algorithm [9], that runs in $O\left(n^{\omega}\right)$ time, and the orthogonal algorithm [7], that runs in $O\left(n^{\omega+1}\right)$ time, where $\omega$ is the matrix multiplication exponent. Despite being faster, the $M_{I}$ algorithm always produces the same median for a given input triple, whereas the orthogonal algorithm, being randomized, can produce any of the orthogonal medians in situations where multiple solutions exist. Besides, the $M_{I}$ algorithm works only for genomic matrices, while the orthogonal algorithm is more general, accepting orthogonal matrices as inputs.

\section{The Orthogonal Algorithm and Its Modification}

We use the following matrix notation throughout the remainder of the paper. Vectors in $\mathbb{R}^{n}$ are written as $n \times 1$ matrices, that is, column vectors. An $m \times n$ matrix $A$ can be then seen as the linear mapping $A: \mathbb{R}^{n} \mapsto \mathbb{R}^{m}$ taking $x \in \mathbb{R}^{n}$ to $A x \in \mathbb{R}^{m}$, where $A x$ is matrix multiplication. When we talk about the image $\operatorname{im}(A)$ of $A$ and the $\operatorname{kernel} \operatorname{ker}(A)$ of $A$, we are referring to the image and the kernel of this linear mapping. We also extend matrix multiplication to sets. If $M$ is a matrix and $V$ is a set of vectors, then

$$
M V=\{M v \mid v \in V\} .
$$

In addition, while in our main motivating application the sizes of the genome matrices are even (with each region having a tail and a head), nothing in what follows uses this fact, and the results are valid for odd-sized square matrices as well.

We define a generalized median of three matrices $A, B$, and $C$ to be a matrix $M$ that minimizes

$$
d(A, M)+d(B, M)+d(C, M)
$$

where $d(X, Y)$ is the rank of the difference $X-Y$. We call it generalized median as opposed to just median to emphasize the fact that the matrices here do not have to be genomic or orthogonal: both the inputs $A, B, C$ and the solution $M$ can be general matrices. 
We briefly present the $O\left(n^{\omega+1}\right)$ orthogonal algorithm for computing a generalized median of 3 orthogonal matrices as published in 2018 [7]. The idea is to "walk towards the median", as follows. Start with orthogonal matrices $A, B$, and $C$. If $B$ is on a shortest path between $A$ and $C$, meaning that $d(A, B)+d(B, C)=d(A, C)$, then $B$ itself is an orthogonal median that satisfies the lower bound on the total score, given by

$$
\beta(A, B, C):=\frac{1}{2} d(A, B)+d(B, C)+d(C, A) .
$$

Otherwise, we can find a non-zero vector $u \in \operatorname{im}(A-B) \cap \operatorname{im}(C-B)$. With this vector we construct the rank 1 matrix $H=-2 u u^{T} B / u^{T} u$ which, added to $B$, produces another orthogonal matrix $B+H$. Note that $B+H$ is the result of a Householder reflection applied to $B$. We can show that $B+H$ is on a shortest path between $B$ and $A$, and also on a shortest path between $B$ and $C$, and is therefore one step closer to the median than $B$ is. Proceeding in this way, with $B+H$ now replacing $B$, we repeat the procedure until the result is on a shortest path between $A$ and $C$, reaching an orthogonal median $M$ whose score $s(M)$ saturates the lower bound $\beta(A, B, C)$ for the three input matrices. Algorithm 1 below is essentially the same algorithm presented by Chindelevitch et al. [7], where we replaced the recursion by a loop for clarity.

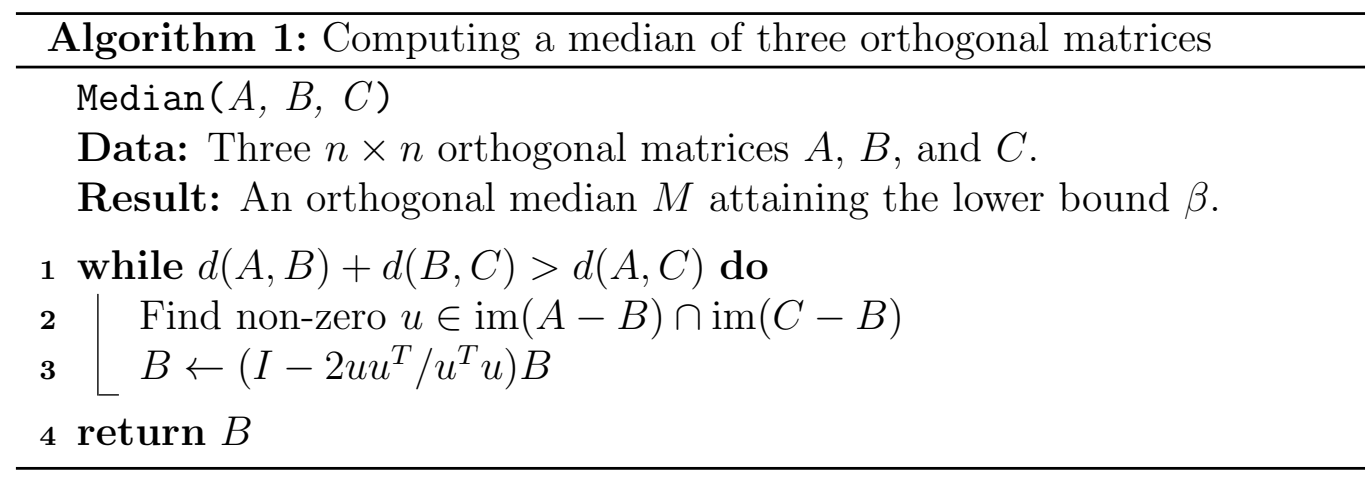

We call any matrix $M$ that lies on a shortest path from $A$ to $B$ an $A B$ intermediate. If a matrix is simultaneously an $A B$ intermediate and a $B C$ intermediate, we call it an $A B C$ double intermediate. It can be shown that Algorithm 1 builds successive $A B C$ double intermediates until a generalized median is reached. Note that a generalized median is an $A B C$ 
double intermediate, but not all $A B C$ double intermediates are generalized medians of $A, B$, and $C$.

Our goal here is to show how to speed up this algorithm if the input matrices are symmetric. We call symmetric, orthogonal matrices SOMs. Given a linear subspace $V$ of $\mathbb{R}^{n}$ and a matrix $M \in \mathbb{R}^{n \times n}$, we say that $V$ is $M$-stable when $M V=V$. Our approach has the following four ingredients:

- We show that the eigenvalues of a SOM can only be equal to +1 or -1 .

- We constructively show that for any $\mathrm{SOM} M$, an $M$-stable space $V$ admits a basis which is composed entirely of eigenvectors of $M$.

- Next, we show that $\operatorname{im}(A-B) \cap \operatorname{im}(C-B)$ is $B$-stable for SOMs $A, B, C$, so we can construct a basis for it consisting only of eigenvectors of $B$.

- Finally, we show that using eigenvectors that are pairwise orthogonal and correspond to the same eigenvalue of $B$ is safe, in the sense that they can all be applied to $B$ (via Householder reflections) simultaneously while remaining an $A B C$ double intermediate.

The following sections are dedicated to each one of these ingredients.

\subsection{A Characterization of the eigenvalues of a SOMs}

Lemma 1. Let $M$ be a SOM. Then all the eigenvalues of $M$ are +1 and -1 .

Proof. Suppose that $M$ is a SOM, and note that $M^{2}=M M^{T}=I$ by symmetry and orthogonality. Since $M$ is a normal matrix, it is diagonalizable by the Spectral Theorem [20], and since $M^{2}=I$, it follows that $M$ only has eigenvalues -1 and 1 .

\subsection{Diagonalization of SOMs On Stable Subspaces}

Before we begin, we review a few key properties of $M$-stability, proved by Chindelevitch et al. [9, Thm.2], that we will need here.

Theorem 1. Let $M$ and $N$ be invertible matrices. Then

a. If $V, W$ are two $M$-stable subspaces, then so are $V \cap W$ and $V+W$.

b. If $M$ is symmetric and $V$ is an $M$-stable subspace, then so is $V^{\perp}$. 
c. If $M^{2}=I=N^{2}$ then the subspace $\operatorname{ker}(M-N)$ is $M$-stable and $N$ stable.

Lemma 2. Let $M \in \mathbb{R}^{n \times n}$ be a $S O M$ and let $V \subseteq \mathbb{R}^{n}$ be a vector subspace. Then $V$ is $M$-stable if and only if $V$ has a basis formed by eigenvectors of $M$.

Proof. One direction is obvious. Let $d$ be the dimension of $V$ and $\left\{u_{1}, \ldots, u_{d}\right\}$, a basis of $V$ such that $M u_{i}=\lambda_{i} u_{i}$ (where $\lambda_{i} \in\{-1,1\}$ by Lemma 1 ), then

$$
v \in V \Longrightarrow v=\sum_{i=1}^{d} \alpha_{i} u_{i} \Longrightarrow M v=\sum_{i=1}^{d} \alpha_{i} M u_{i}=\sum_{i=1}^{d} \alpha_{i} \lambda_{i} u_{i} \in V
$$

so $M V \subseteq V$ and, as $M$ is invertible (by orthogonality), $V$ is $M$-stable.

For the other direction, the proof is by strong induction on $d=\operatorname{dim}(V)$. The statement is obvious for $d \leq 1$. Suppose $d>1$ and the statement is true for all dimensions less than $d$. Let $u_{1}$ be a non-zero vector in $V$, and define $v_{1}:=M u_{1}$. Note that $v_{1} \neq 0$ since $M$ is invertible.

First, if $v_{1}$ is a multiple of $u_{1}$, say $v_{1}=\lambda_{1} u_{1}$, then $u_{1}$ is an eigenvector of $M$. Let $V_{1}$ denote the 1 -dimensional span of $u_{1}$, which is obviously $M$ stable. Then $V$ is the direct sum of $V_{1}$ and $V \cap V_{1}^{\perp}$. The latter is $M$-stable by properties (a) and (b) of Theorem 1 , and since its dimension is $d-1$, it has a basis containing $d-1$ eigenvectors of $M$ by the induction hypothesis. This basis, together with $u_{1}$, is the desired basis of $V$.

Second, if $v_{1}$ is not a multiple of $u_{1}$, we note that $x_{1}:=u_{1}+v_{1}$ is a 1 eigenvector of $M$ while $y_{1}:=u_{1}-v_{1}$ is a -1 eigenvector of $M$. Since $u_{1}$ and $v_{1}$ are linearly independent, there is a basis of $V$ that contains both of them. Let $V_{2}$ denote the 2-dimensional span of $u_{1}$ and $v_{1}$, which is also the span of $x_{1}$ and $y_{1}$. Then $V$ is the direct sum of $V_{2}$ and $V \cap V_{2}^{\perp}$. The latter is $M$-stable by properties (a) and (b) of Theorem 1 , and since its dimension is $d-2$, it has a basis containing $d-2$ eigenvectors of $M$ by the induction hypothesis. This basis, together with $x_{1}$ and $y_{1}$, is the desired basis of $V$.

\subsection{The $B$-stability of $\operatorname{im}(A-B) \cap \operatorname{im}(C-B)$ for $S O M$ s $A, B, C$}

Lemma 3. Let $A, B, C \in \mathbb{R}^{n \times n}$ be SOMs. Then $\operatorname{im}(A-B) \cap \operatorname{im}(C-B)$ is B-stable.

Proof. This is immediate from Theorem 1. First, $\operatorname{ker}(A-B)=\{x \mid A x=B x\}$ is $B$-stable by part (c), since $A^{2}=I=B^{2}$. Similarly, $\operatorname{ker}(C-B)$ is $B$-stable 
by part (c). The symmetry of $A, B, C$ then implies, by part (b), that both $\operatorname{im}(A-B)=\operatorname{ker}(A-B)^{\perp}$ and $\operatorname{im}(C-B)=\operatorname{ker}(C-B)^{\perp}$ are $B$-stable. Finally, their intersection $\operatorname{im}(A-B) \cap \operatorname{im}(C-B)$ is $B$-stable by part (a).

\subsection{Simultaneous Application of an Entire Eigenspace}

The main challenge of Algorithm 1 was the requirement to recompute a new basis for $\operatorname{im}(A-B) \cap \operatorname{im}(C-B)$ every time $B$ changed via the Householder reflection $B \rightarrow B-2 u u^{T} B / u^{T} u$. As we now show, this challenge can be overcome for SOMs by grouping together the basis vectors of $B$ to the same eigenvalue.

For this, we first need a few definitions and a theorem. We say that an $n \times n$ matrix $H$ decomposes another $n \times n$ matrix $F$ whenever $r(H)+r(F-$ $H)=r(F)$, where $r(\cdot)$ is the rank function. Recall that a projection is a square matrix $P$ satisfying $P^{2}=P$.

Theorem 2. If $P$ is a projection such that $\operatorname{im}(P) \subseteq \operatorname{im}(F)$, then $P F$ decomposes $F$.

Proof. We will first show that $\operatorname{im}(P F) \cap \operatorname{im}(F-P F)=\{0\}$. Indeed, if

$$
x=P F y=(F-P F) z,
$$

then

$$
x=P F y=P P F y=P(F-P F) z=P F z-P F z=0 .
$$

Hence the subspace $\operatorname{sum} \operatorname{im}(P F) \oplus \operatorname{im}(F-P F)$ is a direct sum and its dimension is the sum of the dimensions of its summands. On the other hand, both $\operatorname{im}(P F)$ and $\operatorname{im}(F-P F)$ are contained in $\operatorname{im}(F)$, and therefore

$\operatorname{dimim}(P F)+\operatorname{dimim}(F-P F)=\operatorname{dim}(\operatorname{im}(P F) \oplus \operatorname{im}(F-P F)) \leq \operatorname{dim} \operatorname{im}(F)$, or,

$$
r(P F)+r(F-P F) \leq r(F) .
$$

But we also have $r(F)=r(P F)+(F-P F) \leq r(P F)+r(F-P F)$ by the properties of ranks. We therefore conclude that

$$
r(P F)+r(F-P F)=r(F)
$$

that is, $P F$ decomposes $F$. 
We now show that, under certain circumstances, it is safe to apply multiple Householder reflections simultaneously. This is the content of the following theorem, which can be used to generalize a result from the Additional File 1 of Childelevitch et al. [7]. Notice that the existence of the matrix $U$ in the theorem is guaranteed by Lemmas 2 and 3.

Theorem 3. If $A, B \in \mathbb{R}^{n \times n}$ are $S O M$ s, and $U$ is an $n \times h$ matrix consisting of orthonormal eigenvectors of $B$ to the same eigenvalue, with $\operatorname{im}(U) \subseteq$ $\operatorname{im}(B-A)$, then $H:=2 U U^{T} B$ decomposes $B-A$.

Proof. Since $\operatorname{im} U \subseteq \operatorname{im}(B-A)$, each column of $U$ can be written as a linear combination of the columns of $B-A$, i.e.

$$
U=(B-A) X
$$

for some $n \times h$ matrix $X$. Transposing and multiplying both sides by $B$ on the right, and using the orthogonality of $A$ and $B$, we can write

$U^{T} B=X^{T}\left(B^{T}-A^{T}\right) B=X^{T}(I-A B)=X^{T}(A A-A B)=X^{T} A(A-B)$.

By combining this with the original definition of $X$, we get

$$
H=2 U U^{T} B=2(B-A) X X^{T} A(A-B)=-2(B-A) X X^{T} A(B-A) .
$$

In view of Theorem 2 , all we need is to prove that $-2(B-A) X X^{T} A$ is a projection, since its image is contained $\operatorname{in} \operatorname{im}(B-A)$ because it is a product that starts with $B-A$ as a factor.

By the hypothesis on $U$ and the orthogonality of $A$ and $B$, we now get

$$
\begin{aligned}
I & =U^{T} U=[(B-A) X]^{T}(B-A) X= \\
& =X^{T}\left(B^{T}-A^{T}\right)(B-A) X= \\
& =X^{T}(2 I-B A-A B) X .
\end{aligned}
$$

If we were able to show that

$$
X^{T} B A X=X^{T} A B X,
$$

it would follow that

$$
\begin{aligned}
{\left[-2 X^{T} A\right][(B-A) X] } & =-2 X^{T} A(B-A) X= \\
& =-2 X^{T} A B X+2 X^{T} A A X= \\
& =X^{T}(2 I-B A-A B) X=I,
\end{aligned}
$$


which would imply that multiplying the two factors between brackets in the other order, that is, $[(B-A) X]\left[-2 X^{T} A\right]$, is a projection, exactly what we need. This follows from $M N=I \Longrightarrow(N M)^{2}=N(M N) M=N M$.

In general, equation (1) is false (see the counterexample below). However, we will show that if $U$ contains eigenvectors of $B$ to the same eigenvalue, it holds, and thus $H$ decomposes $B-A$. First, note that, by the definition of $U$, we have $A X=B X-U$, so equation (1) is equivalent to

$$
\begin{aligned}
& X^{T} B A X=X^{T} A B X \Longleftrightarrow X^{T} B(B X-U)=(B X-U)^{T} B X \Longleftrightarrow \\
& X^{T} B^{T} B X-X^{T} B U=X^{T} B^{T} B X-U^{T} B X \Longleftrightarrow X^{T} B U=U^{T} B X .
\end{aligned}
$$

Now suppose that each column of $U$ is an eigenvector of $B$, i.e. $B U=U \Lambda$, where $\Lambda$ is diagonal. Then $B^{T} U=B^{-1} U=U \Lambda^{-1}$ and the condition becomes $X^{T} B U=U^{T} B X \Longleftrightarrow X^{T} B^{-1} U=\left(B^{-1} U\right)^{T} X \Longleftrightarrow X^{T} U \Lambda^{-1}=\Lambda^{-1} U^{T} X$,

where we used the fact that $\Lambda$ is diagonal. If all the eigenvalues are also equal, then $\Lambda=\lambda I$, and the condition reduces to

$$
X^{T} U=U^{T} X \Longleftrightarrow X^{T}(B-A) X=X^{T}(B-A)^{T} X=X^{T}\left(B^{T}-A^{T}\right) X,
$$

which is true by the symmetry of $A$ and $B$, completing the proof.

Counterexample: Here is an example when the theorem's conclusion is false because the eigenvalues are different and equation (1) fails.

Let $A=e_{1} e_{2}^{T}+e_{2} e_{1}^{T}+e_{3} e_{3}^{T}$ and $B=e_{1} e_{3}^{T}+e_{2} e_{2}^{T}+e_{3} e_{1}^{T}$, where $e_{1}, e_{2}$, and $e_{3}$ are the standard basis of $\mathbb{R}^{3}$. We will take the two orthogonal unit vectors $u_{1}=\frac{1}{\sqrt{2}}[1,0,-1]^{T}$ and $u_{2}=\frac{1}{\sqrt{6}}[1,-2,1]^{T}$, which are in $\operatorname{im}(B-A)$ as their entries add up to 0 . Note that $B u_{1}=-u_{1}$ and $B u_{2}=u_{2}$, so both are eigenvectors of $B$, but to different eigenvalues. We also define $U:=\left[u_{1} u_{2}\right]$ so $U^{T} U=I_{2}$.

Even though both $B-2 u_{1} u_{1}^{T} B=I$ and $B-2 u_{2} u_{2}^{T} B$ are intermediates between $A$ and $B$, the matrix

$$
B-2 U U^{T} B=B-2 u_{1} u_{1}^{T} B-2 u_{2} u_{2}^{T} B=I-2 u_{2} u_{2}^{T}=\frac{1}{3}\left[\begin{array}{rrr}
2 & 2 & -1 \\
2 & -1 & 2 \\
-1 & 2 & 2
\end{array}\right]
$$

is in fact at a rank distance of 2 from both $A$ and $B$, and therefore $2 U U^{T} B$ does not decompose $B-A$. 
Corollary 1. If $A, B, C \in \mathbb{R}^{n \times n}$ are $S O M$ s and $U$ is an $n \times h$ matrix consisting of orthonormal eigenvectors of $B$ to the eigenvalue \pm 1 , with $\operatorname{im}(U) \subseteq$ $\operatorname{im}(B-A) \cap \operatorname{im}(B-C)$, then $B^{\prime}:=B \mp 2 U U^{T}$ is an $A B C$ double intermediate.

Proof. Since $U$ consists of eigenvectors of $B$ to the same eigenvalue, say $\lambda$, we have $B U=\lambda U$, and thus $U^{T} B=\lambda U^{T}$ and $U U^{T} B=\lambda U U^{T}$. Thus, since $\lambda=1$ or $\lambda=-1$ by Corollary 1 , we have that $\pm 2 U U^{T}$ decomposes both $B-A$ and $B-C$ by Theorem 3. Therefore, $B^{\prime}=B \mp 2 U U^{T}$ is an $A B C$ double intermediate.

\subsection{Closing Argument}

Now that we have established that an orthogonal set of eigenvectors of $B$ to the same eigenvalue can be used as a multi-step in the walk towards a median, we need one last result showing that after the first multi-step, all the remaining steps are automatically eigenvectors of $B$ to the other eigenvalue.

Lemma 4. Let $A, B, C \in \mathbb{R}^{n \times n}$ be $S O M$ s and $\lambda \in\{-1,1\}$. Let $U$ consist of an orthonormal basis of $\operatorname{im}(B-A) \cap \operatorname{im}(B-C) \cap \operatorname{ker}(B-\lambda I)$, i.e. the set of all vectors in $\operatorname{im}(B-A) \cap \operatorname{im}(B-C)$ that are eigenvectors of $B$ to the eigenvalue $\lambda$. Let $B^{\prime}=B-2 \lambda U U^{T}$. Then $\operatorname{im}\left(B^{\prime}-A\right) \cap \operatorname{im}\left(B^{\prime}-C\right) \subseteq \operatorname{ker}\left(B^{\prime}-(-\lambda) I\right)$.

Proof. We reason as follows. First, since $\operatorname{im}(U) \subseteq \operatorname{im}(B-A)$, say $U=$ $(B-A) W$, we have that $\operatorname{im}\left(B^{\prime}-A\right)=\operatorname{im}\left(B-A-2 \lambda U U^{T}\right)=\operatorname{im}((B-$ $\left.A)\left(I-2 \lambda W W^{T}(B-A)\right)\right) \subseteq \operatorname{im}(B-A)$. Similarly, $\operatorname{im}\left(B^{\prime}-C\right) \subseteq \operatorname{im}(B-C)$. It follows that

$$
\operatorname{im}\left(B^{\prime}-A\right) \cap \operatorname{im}\left(B^{\prime}-C\right) \subseteq \operatorname{im}(B-A) \cap \operatorname{im}(B-C) .
$$

Furthermore, by the symmetry of $B$ and since $B U=\lambda U$, we have

$$
\left(B^{\prime}\right)^{T}=B^{T}-2 \lambda U U^{T}=B^{\prime}
$$

and

$$
\begin{aligned}
\left(B^{\prime}\right)^{2} & =\left(B-2 \lambda U U^{T}\right)\left(B-2 \lambda U U^{T}\right) \\
& =I-2 \lambda B U U^{T}-2 \lambda U U^{T} B+4 \lambda^{2} U U^{T} U U^{T} \\
& =I-2 \lambda^{2} U U^{T}-2 \lambda^{2} U U^{T}+4 \lambda^{2} U U^{T} \\
& =I,
\end{aligned}
$$


so $B^{\prime}$ is also a SOM. By Lemma 3, we know that $\operatorname{im}\left(B^{\prime}-A\right) \cap \operatorname{im}\left(B^{\prime}-C\right)$ is $B^{\prime}$-stable, and thus, admits an orthonormal basis $\mathcal{B}$ consisting of eigenvectors of $B^{\prime}$ by Lemma 2 .

Suppose that $u$ is one of those basis vectors. We know that $u \in \operatorname{im}(B-$ $A) \cap \operatorname{im}(B-C)$. Let us decompose the space $\operatorname{im}(B-A) \cap \operatorname{im}(B-C)$ into the direct sum of its intersection with the $\lambda$-eigenspace of $B$, spanned by the columns of $U$ by construction, and its intersection with the $-\lambda$-eigenspace of $B$, spanned, say, by the columns of $V$. This is possible by Lemmas 2 and 3. Thanks to the symmetry of $B$, we know that the column spaces of $U$ and $V$ are orthogonal, so $U^{T} V=0$, and the sum is an orthogonal one. We also have $u=U x+V y$ for some $x$ and $y$. Now

$$
\begin{aligned}
B^{\prime} u & =B^{\prime}(U x+V y) \\
& =B(U x+V y)-2 \lambda U U^{T}(U x+V y) \\
& =\lambda U x-\lambda V y-2 \lambda U x-0 \\
& =-\lambda u,
\end{aligned}
$$

meaning that all the vectors in $\mathcal{B}$ are eigenvectors of $B^{\prime}$ to the eigenvalue $-\lambda$. This concludes the proof.

Lemma 5. Let $A, B, C \in \mathbb{R}^{n \times n}$ be $S O M s$, and suppose that there exists a matrix $U$ consisting of an orthonormal basis of $\operatorname{im}(B-A) \cap \operatorname{im}(B-C)$ composed entirely of eigenvectors of $B$ to the same eigenvalue $\lambda$. Then, $M:=B-2 \lambda U U^{T}$ is a generalized median of $A, B, C$.

Proof. Let $d:=\operatorname{dim}(\operatorname{im}(B-A) \cap \operatorname{im}(B-C))$ be the number of columns of $U$, so that $U$ is an $n \times d$ matrix with $U^{T} U=I_{d}$. By Corollary 1 , we know that $M=B-2 \lambda U U^{T}$ is an $A B C$ double intermediate. It follows that

$$
d(A, M)+d(M, B)=d(A, B), d(C, M)+d(M, B)=d(C, B) .
$$

Now consider $d(A, M)+d(M, C)$. From Theorem 1 in [7], we know that

$$
d \geq(d(A, B)+d(B, C)-d(A, C)) / 2
$$

for any orthogonal matrices $A, B, C$. We therefore have

$$
\begin{aligned}
d(A, M)+d(M, C) & =(d(A, B)-d)+(d(B, C)-d) \\
& =d(A, B)+d(B, C)-2 d \\
& \leq d(A, C),
\end{aligned}
$$


which must therefore be an equality. It follows that $M$ is indeed a generalized median of $A, B$ and $C$.

We state one final result, which is essentially identical to Theorem 4 in $[7]$.

Lemma 6. Let $N$ be an $A B C$ double intermediate and let $M$ be a generalized median of $A, N$ and $C$. Then $M$ is also a generalized median of $A, B$ and $C$.

\subsection{The Improved Algorithm}

The modified algorithm is then as follows:

- Compute an orthonormal basis of $\operatorname{im}(A-B) \cap \operatorname{im}(C-B)$ consisting entirely of eigenvectors of $B$ (this is possible by Lemmas 2 and 3 ).

- Let $X$ contain, as its columns, all the basis vectors to the eigenvalue 1 and replace $B$ with $B^{\prime}=B-2 X X^{T}$. Then $B^{\prime}$ is an $A B C$ double intermediate by Corollary 1 , and we want a generalized median of $A, B^{\prime}$ and $C$ by Lemma 6 .

- Recompute an orthonormal basis of $\operatorname{im}\left(A-B^{\prime}\right) \cap \operatorname{im}\left(C-B^{\prime}\right)$; by Lemma 4 , it will consist entirely of eigenvectors of $B^{\prime}$ to the eigenvalue -1 , so putting them into a matrix $Y$ as columns, we get $B^{\prime \prime}=B^{\prime}+2 Y Y^{T}$, which is a generalized median of $A, B$ and $C$ by Lemmas 5 and 6 .

\subsection{Running-time analysis}

Since each step takes $O\left(n^{\omega}\right)$ time in the worst case, the full algorithm also takes $O\left(n^{\omega}\right)$ time. Note also that we know exactly how many vectors we'll need in step 3 (the difference between the number of vectors in step 1 and the distance from $B$ to any generalized median), but it does not appear to be straightforward to figure out precisely which ones of those will yield the result.

Note lastly that the algorithm establishes that any three SOMs admit a generalized median which is an SOM.

A previous algorithm, the $M_{I}$ algorithm, also outputs a symmetric, orthogonal median in time $O\left(n^{\omega}\right)[9]$. However, it requires the inputs to be genomic, whereas the algorithm we just presented works for any symmetric 
orthogonal inputs. Furthermore, even for genomic matrices (which are automatically symmetric and orthogonal), the output of the two algorithms is not always the same. For instance, consider $A, B$, and $C$ given by:

$$
\left[\begin{array}{llllll}
0 & 1 & 0 & 0 & 0 & 0 \\
1 & 0 & 0 & 0 & 0 & 0 \\
0 & 0 & 0 & 0 & 1 & 0 \\
0 & 0 & 0 & 1 & 0 & 0 \\
0 & 0 & 1 & 0 & 0 & 0 \\
0 & 0 & 0 & 0 & 0 & 1
\end{array}\right],\left[\begin{array}{llllll}
0 & 0 & 1 & 0 & 0 & 0 \\
0 & 0 & 0 & 1 & 0 & 0 \\
1 & 0 & 0 & 0 & 0 & 0 \\
0 & 1 & 0 & 0 & 0 & 0 \\
0 & 0 & 0 & 0 & 1 & 0 \\
0 & 0 & 0 & 0 & 0 & 1
\end{array}\right],\left[\begin{array}{llllll}
0 & 0 & 0 & 0 & 0 & 1 \\
0 & 1 & 0 & 0 & 0 & 0 \\
0 & 0 & 1 & 0 & 0 & 0 \\
0 & 0 & 0 & 0 & 1 & 0 \\
0 & 0 & 0 & 1 & 0 & 0 \\
1 & 0 & 0 & 0 & 0 & 0
\end{array}\right],
$$

respectively. For these matrices, $M_{I}=I$ (the identity matrix), whereas the SOM algorithm yields something very different:

$$
M=\frac{1}{7}\left[\begin{array}{rrrrrr}
3 & 4 & 4 & -2 & -2 & 0 \\
4 & 3 & -4 & 2 & 2 & 0 \\
4 & -4 & 3 & 2 & 2 & 0 \\
-2 & 2 & 2 & -1 & 6 & 0 \\
-2 & 2 & 2 & 6 & -1 & 0 \\
0 & 0 & 0 & 0 & 0 & 7
\end{array}\right]
$$

We thus have two different algorithms with the same complexity, one more general than the other, that may find distinct solutions on the same inputs.

\section{Extending the Algorithm to Permutations}

Several pieces in the above strategy seem to generalize to permutations, or even to generic orthogonal matrices. We outline the main ones below.

\subsection{Characterization of Orthogonal Idempotent Matrices}

We would like to be able to say something similar to what we know to be true for SOMs, namely, that any real orthogonal matrix $M$ with $M^{k}=I$ for some integer $k$ is orthogonally similar to a permutation matrix. Unfortunately, this is not the case, as shown in the following example:

$$
A=\left[\begin{array}{rrr}
0 & 0 & -1 \\
1 & 0 & 0 \\
0 & 1 & 0
\end{array}\right]
$$

The matrix $A$ is the companion matrix of the polynomial $p(x):=x^{3}+1$, so its eigenvalues are the odd powers of the sixth root of unity; if $w:=\exp (2 \pi i / 6)$, 
then the spectrum of $A$ is $\left\{w, w^{3}, w^{5}\right\}$. It is therefore easy to check that $A^{6}=I$, and $k=6$ is the lowest power that works. However, any permutation with eigenvalues $w, w^{3}, w^{5}$ must also have eigenvalues $w^{0}, w^{2}, w^{4}$, which $A$ lacks, so it is not similar (orthogonally or otherwise) to any permutation.

Note that the dimension of $A$ can be made arbitrarily large by using a block-diagonal matrix with $A$ in each diagonal block (in other words, replacing $A$ with $I_{m} \otimes A$, where $\otimes$ is the Kronecker product), so requiring $k \leq n$ does not help.

\subsection{M-stability For Orthogonal Matrices}

Surprisingly, the following generalization of part (b) of Theorem 1 holds for arbitrary orthogonal matrices.

Lemma 7. Let $M$ be orthogonal and let $V$ be an $M$-stable space. Then $V^{\perp}$ is also $M$-stable.

Proof. Let $v \in V^{\perp}$. We need to show that $M v \in V^{\perp}$. By hypothesis, $M V=V$, which also means that $V=M^{-1} V$. Let $w \in V$ be arbitrary, and let $z=M^{-1} w \in V$. We have

$$
(M v)^{T} w=v^{T} M^{T} w=v^{T} M^{-1} w=v^{T} z=0 .
$$

Since $w$ was chosen arbitrarily, we conclude that $M v \in V^{\perp}$, so $V^{\perp}$ is indeed $M$-stable.

However, this is not sufficient to prove that $\operatorname{im}(B-A)$ is $B$-stable, which is in fact false, even for permutations. For instance, consider $A$ and $B$ defined as follows:

$$
\begin{aligned}
& A e_{1}=e_{1}, B e_{1}=e_{2} \\
& A e_{2}=e_{3}, B e_{2}=e_{3} \\
& A e_{3}=e_{4}, B e_{3}=e_{1} \\
& A e_{4}=e_{2}, B e_{4}=e_{4}
\end{aligned}
$$

Then the space $\operatorname{im}(B-A)$ is spanned by $u_{1}=e_{1}-e_{4}$ and $u_{2}=e_{2}-e_{4}$, of which the latter maps to $u_{3}=e_{3}-e_{4}$ by $B$, and $u_{3} \notin \operatorname{im}(B-A)$.

We already know that the $B$-stability result is true if $B-A$ is symmetric. Unfortunately, this can only happen for permutations $A$ and $B$ if both are involutions; to see this, note that $B-A$ has 1 entries from $B$ and -1 entries from $A$, so the only way to ensure its symmetry is to have $A=A^{T}$ and $B=B^{T}$.

On the positive side, the $B$-stability result is also true for a pair $A$ and $B$ that commutes. This is the subject of the next lemma. 
Lemma 8. Suppose that $A$ and $B$ are commuting matrices; then $\operatorname{im}(B-A)$ is B-stable.

Proof. Let $x \in \operatorname{im}(B-A)$, say, $x=(B-A) u$. We want to show that $B x \in \operatorname{im}(B-A)$. We have

$B x=B(B-A) u=\left(B^{2}-B A\right) u=\left(B^{2}-A B\right) u=(B-A) B u \in \operatorname{im}(B-A)$.

One possible idea that this result suggests is to multiply all the inputs by an orthogonal matrix on the right to go from $A, B, C$ to $A U, B U, C U$, such that both $A U$ and $C U$ commute with $B U$. This is easily achieved by using $U=B^{T}$; however, as we shall see further in this section, $M$-stability does not always suffice for a set of orthonormal eigenvectors to the same eigenvalue to be "safe".

\subsection{Eigenbases For M-stable Spaces With M Orthogonal}

Suppose that $M$ is orthogonal and $V$ is an $M$-stable space. This is sufficient for $V$ to have a basis consisting of eigenvectors of $M$. First, recall that, by Lemma $7, V^{\perp}$ is $M$-stable as well.

Lemma 9. Let $M$ be an orthogonal matrix and let $V$ be an $M$-stable space. Then $V$ admits a basis consisting of eigenvectors of $M$.

Proof. Let $U$ be a matrix whose columns form an orthonormal basis of $V$. By orthogonality of $M$, we have that the columns of $M U$ also form an orthonormal basis of $V$ because they are unit vectors (as $M$ preserves norms), pairwise orthogonal (as $M$ preserves angles) and linearly independent (as $M$ is invertible). Therefore, we can write

$$
M U=U A
$$

for some $k \times k$ matrix $A$, where $k=\operatorname{dim}(V)$ is the number of columns of $U$. Now $A=U^{T} M U$ and $A A^{T}=U^{T} M U U^{T} M^{T} U$. We claim that $M U U^{T}=$ $U U^{T} M$. In fact, $U U^{T}$ is just the orthogonal projection onto $V$. If $v \in V$, then $M U U^{T} v=M v=U U^{T} M v$, since $M v \in V$ as $V$ is $M$-stable. If $v \in V^{\perp}$, then $M U U^{T} v=M 0=0=U U^{T} M v$ since $M v \in V^{\perp}$ as well by Lemma 7 . As $U U^{T} M$ and $M U U^{T}$ agree on both $V$ and $V^{\perp}$, they must be equal. 
It follows that

$$
A A^{T}=U^{T} M U U^{T} M^{T} U=U^{T} U U^{T} M M^{T} U=U^{T} U U^{T} U=I,
$$

so $A$ is orthogonal, and therefore orthogonally diagonalizable by the Spectral theorem [20]. Let us write $A=X D X^{T}$, with $X$ orthogonal and $D$ diagonal, so that $A X=X D$ and $M U X=U X D$. Therefore the columns of $U X$ consitute a basis of $V$ which consists of eigenvectors of $M$.

In general, this construction of a basis is rather complex, and may require arbitrary matrix operations. However, the case of permutations is a lot easier and more similar to that of SOMs; in particular, we can start from an arbitrary vector in the space and use it to get a basis. We have the following

Lemma 10. Let $M$ be a permutation matrix and let $V$ be an $M$-stable space. Then $V$ admits an explicit basis consisting of eigenvectors of $M$.

Proof. We prove the statement by strong induction on the dimension of $V$. The base case is zero-dimensional or one-dimensional spaces, for which the statement is obviously true.

Let $V$ be an $M$-stable space, and let $k$ be such that $M^{k}=I$ (we can take $k$ to be the least common multiple of all the cycle lengths of the permutation corresponding to $M$ ). The statement is trivial for $k=1$, so we can assume that $k>1$. Let $u \in V$ be any non-zero vector. By $M$-stability, we have $M^{m} u \in V$ for any natural number $m$. Let $j$ be the smallest positive integer for which $M^{j+1} u=u$; such a $j$ is guaranteed to exist because $M^{k} u=u$. Consider the set of vectors $S_{u}:=\left\{u, M u, \ldots, M^{j} u\right\}$. By construction, they are guaranteed to be distinct; otherwise, if $M^{\alpha} u=M^{\beta} u$ with $\alpha \neq \beta$, then $M^{|\alpha-\beta|} u=u$, and $0<|\alpha-\beta| \leq j$.

Let $\omega_{j+1}=\exp (2 \pi i /(j+1))$ be the $(j+1)$-th root of unity. For $0 \leq s \leq j$, consider the vector

$$
v[u, s]:=\sum_{\ell=0}^{j} \omega_{j+1}^{\ell s} M^{\ell} u .
$$

We claim that the $v[u, s]$ are eigenvectors of $M$ and that they have the same span as $S_{u}$. 
For the first statement, note that

$$
\begin{aligned}
M v[u, s] & =M \sum_{\ell=0}^{j} \omega_{j+1}^{\ell s} M^{\ell} u \\
& =\sum_{\ell=0}^{j} \omega_{j+1}^{\ell s} M^{\ell+1} u \\
& =\omega_{j+1}^{-s} \sum_{\ell=0}^{j} \omega_{j+1}^{(\ell+1) s} M^{\ell+1} u \\
& =\omega_{j+1}^{-s} v[u, s],
\end{aligned}
$$

where the last equality follows from the fact that

$$
\sum_{\ell=0}^{j} \omega_{j+1}^{(\ell+1) s} M^{\ell+1} u=\sum_{\ell=0}^{j-1} \omega_{j+1}^{(\ell+1) s} M^{\ell+1} u+\omega_{j+1}^{(j+1) s} M^{j+1} u=\sum_{\ell=1}^{j} \omega_{j+1}^{\ell s} M^{\ell} u+u .
$$

For the second statement, we note that going from $S_{u}$ to $\{v[u, s]\}_{s=0}^{j}$ is just a discrete Fourier transform [21] on the set $S_{u}$, which is an invertible transformation, so the dimensionality of the underlying space is preserved. Alternatively, one can directly verify that

$$
\sum_{s=0}^{j} \omega_{j+1}^{-\ell s} v[u, s]=(j+1) M^{\ell} u
$$

We note in passing that this reduces to the case of SOMs when we take $k=2$.

Lastly, let us extract a basis from the set $\{v[u, s]\}_{s=0}^{j}$, and call this basis $\mathcal{B}$. Let $V_{u}$ be the span of $\mathcal{B}$. Since $\mathcal{B}$ contains only eigenvectors of $M, V_{u}$ is $M$-stable. Therefore, we also have that $V_{u}^{\perp}$ is $M$-stable by Lemma 7 , and thus the intersection $V \cap V_{u}^{\perp}$ is also $M$-stable. Since its dimension is strictly smaller than the dimension of $V$, it follows by the inductive hypothesis that it has a basis containing only eigenvectors of $M$. Combinining this basis with $\mathcal{B}$ gives such a basis for all of $M$, proving the desired statement.

\subsection{Using Eigenvectors To the Same Eigenvalue}

Unfortunately, even a partial analog of Theorem 3 for general orthogonal matrices or permutations is difficult to prove. The main challenge lies in the condition

$$
V^{T}(B-A) V=V^{T}(B-A)^{T} V
$$


which needs to be satisfied for arbitrary $V$ (the condition on $V$ coming from $U^{T} U=I$ is a weak one), and this should not happen unless $B-A$ is symmetric.

\section{Conclusions}

We have shown how to speed up the orthogonal algorithm for finding a generalized median of three symmetric, orthogonal matrices so that it runs in $O\left(n^{\omega}\right)$ time, where $\omega$ is the matrix multiplication exponent, an improvement over the originally proposed algorithm running in $O\left(n^{\omega+1}\right)$, where $n$ is the number of conserved regions among the genomes.

In closing, we would like to suggest two open questions to be explored in future research:

- Can generalized medians be computed faster than $O\left(n^{\omega}\right)$ if the inputs are either general or symmetric permutations?

- Can we show that some of the available algorithms for generalized medians actually find a genome (a symmetric permutation matrix) if there exists one that saturates the bound (i.e. scores exactly $\beta(A, B, C))$ ?

\section{Acknowledgments}

Funding: This work was supported by the State of Sao Paulo Research Foudation (FAPESP) [grant number 2018/00031-7], as well as the National Science and Engineering Research Council (NSERC) of Canada [grant number RGPIN/04622-2016] and the Sloan Foundation [fellowship number FG2016-6392].

\section{References}

[1] J. Palmer, L. A. Hebron, Plant mitochondrial DNA evolves rapidly in structure, but slowly in sequence, J. Molecular Evolution 28 (1988) 8797.

[2] O. Barış, M. Karadayı, D. Yanmıs, M. Gülüce, Genomic rearrangements and evolution, in: M. Silva-Opps (Ed.), Current Progress in Biological Research, IntechOpen, 2013, pp. 19-39. doi:10.5772/55456. 
[3] O. O. Bochkareva, E. V. Moroz, I. I. Davydov, M. S. Gelfand, Genome rearrangements and selection in multi-chromosome bacteria Burkholderia spp., BMC Genomics 19 (1) (2018) 965.

[4] M. Hoeksema, M. J. Jonker, K. Bel, S. Brul, B. H. Ter Kuile, Genome rearrangements in Escherichia coli during de novo acquisition of resistance to a single antibiotic or two antibiotics successively, BMC Genomics 19 (1) (2018) 973.

[5] K. M. Swenson, M. Blanchette, Large-scale mammalian genome rearrangements coincide with chromatin interactions, Bioinformatics 35 (14) (2019) i117-i126. doi:10.1093/bioinformatics/btz343.

[6] P. Simonaitis, A. Chateau, K. M. Swenson, A general framework for genome rearrangement with biological constraints, Algorithms for Molecular Biology 14 (15) (2019) 1-14.

[7] L. Chindelevitch, J. P. P. Zanetti, J. Meidanis, On the rank-distance median of 3 permutations, BMC Bioinformatics 19 (Suppl 6) (2018) 142 .

[8] J. P. P. Zanetti, P. Biller, J. Meidanis, Median approximations for genomes modeled as matrices, Bulletin of Mathematical Biology 78 (4) (2016) 786-814, preliminary version On the matrix median problem in: Proc. 13th WABI, pp. 230-243. Springer, 2013.

[9] L. Chindelevitch, S. La, J. Meidanis, A cubic algorithm for the generalized rank median of three genomes, Algorithms for Molecular Biology 14 (16) (2019) 23 pages, preliminary version in: 16th RECOMB-CG Workshop in 2018.

[10] J. P. P. Zanetti, L. Chindelevitch, J. Meidanis, Counting sorting scenarios and intermediate genomes for the rank distance, in: I. Holmes, C. Martín-Vide, M. A. Vega-Rodríguez (Eds.), Algorithms for Computational Biology, Springer International Publishing, Cham, 2019, pp. $137-151$.

[11] J. P. P. Zanetti, L. Chindelevitch, J. Meidanis, Generalizations of the genomic rank distance to indels, in: I. Holmes, C. Martn-Vide, M. VegaRodrguez (Eds.), Algorithms for Computational Biology. AlCoB 2019, 
Vol. 11488 of Lecture Notes in Computer Science, Springer, Cham, 2019, pp. 152-164.

[12] J. H. Nadeau, B. A. Taylor, Lengths of chromosomal segments conserved since divergence of man and mouse, Proceedings of the National Academy of Sciences of the United States of America 81 (3) (1984) 814818, doi:10.1073/pnas.81.3.814.

[13] P. Pevzner, G. Tesler, Genome rearrangements in mammalian evolution: Lessons from human and mouse genomes, Genome Research 13 (1) (2003) 37-45.

[14] Y. Lin, B. M. Moret, A new genomic evolutionary model for rearrangements, duplications, and losses that applies across eukaryotes and prokaryotes, J Comput Biol 18 (9) (2011) 1055-1064.

[15] E. M. Gabidulin, Theory of codes with maximum rank distance, Problems of Information Transmission 21 (1) (1985) 1-12.

[16] J. Meidanis, P. Biller, J. P. P. Zanetti, A matrix-based theory for genome rearrangements, Tech. Rep. IC-18-10, Institute of Computing, University of Campinas (Aug 2018).

[17] N. Saitou, M. Nei, The neighbor-joining method: a new method for reconstructing phylogenetic trees, Mol. Biol. Evol.

[18] R. R. Sokal, P. H. A. Sneath, Numerical taxonomy, W. H. Freeman and Co., San Francisco, 1963.

[19] J. Kováč, B. Brejová, T. Vinař, A practical algorithm for ancestral rearrangement reconstruction, in: T. M. Przytycka, M.-F. Sagot (Eds.), Proceedings of the 11th international conference on Algorithms in Bioinformatics (WABI'11), Vol. 6833 of LNCS, Springer, 2011, pp. 1-13.

[20] S. Axler, Linear Algebra Done Right, 3rd Edition, Undergraduate Texts in Mathematics, Springer, 2015.

[21] G. Strang, Wavelets, American Scientist 82 (3) (1994) 250-255. 